\title{
Coupling of Amino Acids and $\alpha$-Branched Aldehydes
}

\section{Key words}

metal-free

organocatalysis

bioconjugation<smiles>CC(C)C=O</smiles>

(1.0 equiv)

(1.5 equiv)

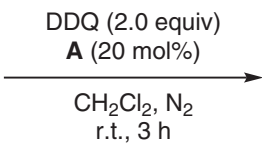<smiles>O=CC([Al])OC(=O)C(P)NC(=O)c1ccccc1</smiles>

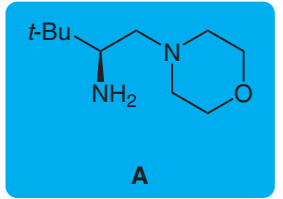<smiles>COc1ccc2cc(C)ccc2c1</smiles><smiles>[Y19][Y4]([H])([H])c1ccc(OCCCCCN)cc1</smiles>

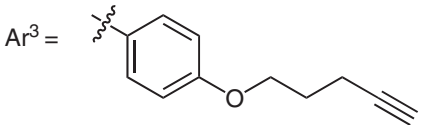<smiles>CC(NC(=O)c1ccccc1)C(=O)O[C@H]([Al])C=O</smiles>

$85 \%$ yield $d r=10: 1^{a}(d r=8: 1)^{b}$

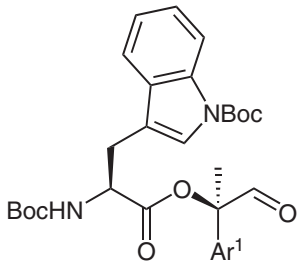

$73 \%$ yield $d r=11: 1^{a}(d r=11: 1)^{b}$<smiles>C[C@H](NC(=O)CNC(=O)OCc1ccccc1)C(=O)O[C@@](C)(Br)C=O</smiles>

$80 \%$ yield $d r=12: 1^{a}(d r=9: 1)^{b}$<smiles>CCCC(NC(=O)c1ccccc1)C(=O)OC(C)C=O</smiles>

$67 \%$ yield $d r=6: 1^{a}(d r=5: 1)^{b}$<smiles>O=CC(OC(=O)c1ccccc1)OC(=O)C(CCCCNC(=O)OCc1ccccc1)NC(=O)c1ccccc1</smiles>

$$
59 \% \text { yield }
$$$$
d r=7: 1^{a}(d r=5: 1)^{b}
$$<smiles>O=CC(O)C(=O)OC(=O)C(CO)NC(=O)c1ccccc1</smiles>

$51 \%$ yield

$$
d r=20: 1^{a}(d r=16: 1)^{b}
$$<smiles>O=CC(O)OC(=O)C(CO)NC(=O)c1ccccc1</smiles>

$86 \%$ yield $d r>20: 1^{a}(d r=12: 1)^{b}$<smiles>O=CC(Br)C(=O)OC(=O)CC(NC(=O)c1ccccc1)C(=O)c1ccccc1</smiles>

$74 \%$ yield $d r=10: 1^{a}(d r=10: 1)^{b}$<smiles>CC(C)(C)OC(=O)N[C@@H](COCc1ccccc1)C(=O)O[C@](C)(C=O)C(=O)c1ccccc1</smiles>

$84 \%$ yield $d r=13: 1^{a}(d r=13: 1)^{b}$

${ }^{a} \mathrm{dr}$ was determined by ${ }^{1} \mathrm{H}$ NMR analysis of isolated product.

${ }^{\mathrm{b}} \mathrm{dr}$ was determined by ${ }^{1} \mathrm{H}$ NMR analysis of crude reaction mixture.

Significance: The synthesis of modified amino acids is significant in the pharmaceutical industry and in medicinal chemistry. The authors used a metal-free oxidative bioconjugation method to couple amino acids and aldehydes.
Comment: Various amino acid esters are synthesized from amino acids and $\alpha$-branched aldehydes. The yields of the reactions were moderate to good, and the stereoselectivities were good. amino acids

aldehydes

asymmetric reaction

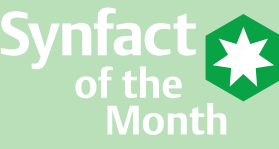

